\title{
Editorial
}

\section{Time-Delay Systems: Modeling, Analysis, Estimation, Control, and Synchronization}

\author{
Olfa Boubaker, ${ }^{1}$ Valentina E. Balas, ${ }^{2}$ Abdellah Benzaouia, ${ }^{3}$ Mohamed Chaabane, \\ Magdi S. Mahmoud, ${ }^{5}$ and Quanmin $\mathrm{Zhu}^{6}$ \\ ${ }^{1}$ National Institute of Applied Sciences and Technology, Tunis, Tunisia \\ ${ }^{2}$ Aurel Vlaicu University, Arad, Romania \\ ${ }^{3}$ Faculty of Sciences Semlalia, Université Cadi Ayyad, Marrakech, Morocco \\ ${ }^{4}$ University of Sfax, Sfax, Tunisia \\ ${ }^{5}$ King Fahd University of Petroleum \& Minerals, Dhahran, Saudi Arabia \\ ${ }^{6}$ Department of Engineering Design and Mathematics, University of the West of England, Bristol, UK
}

Correspondence should be addressed to Olfa Boubaker; olfa.boubaker@insat.rnu.tn

Received 6 March 2017; Accepted 6 March 2017; Published 15 June 2017

Copyright (C) 2017 Olfa Boubaker et al. This is an open access article distributed under the Creative Commons Attribution License, which permits unrestricted use, distribution, and reproduction in any medium, provided the original work is properly cited.

The subject of time-delay systems is a rather old research topic dating back to the works of Euler-Bernoulli in the XVIII century. Effective results in this area were initiated in the late fifties of the twentieth century since the works of Krasovskii and Razumikhin on the Lyapunov functions. However the significant progression in this area has been made during the last decade where numerous books, research, and survey papers and special issues have been devoted.

Delay is not just a mathematical exercise but more importantly is rooted in many natural and man-made systems such as biology, processes industries, and mechatronic motions. In engineering applications, time-delays generally describe propagation phenomena, material or energy transfer in intercommoned systems, and data transmission in communication systems. They have been the main sources inducing oscillations, instability, and poor control performances. Stability analysis and robust control of such systems are then of theoretical and practical importance. Much effort in the analysis and synthesis of these systems has been dedicated to delay-dependent and delay-independent issues based both/either on Lyapunov methods and/or frequency domain techniques. However, it should be noted that there are still numerous challenging issues pending in many classes of time-delay systems.
The purpose of this special issue is to draw attention of the scientific community to some recent advances and possible applications in the analysis, control, and synchronization of time-delay systems remaining unresolved until now. The special issue includes several high-quality papers written by leading and emerging specialists in the field. Engineering applications, such as power systems, rolling mills, hydraulic systems, distillation colons, aircrafts, space launch vehicle, and automotive and robotic systems, are seriously considered in this special issue.

Over 77 submissions from 14 countries (Australia, Brazil, China, Egypt, Iran, Japan, Malaysia, Morocco, Mexico, Nigeria, Republic of Korea, Saudi Arabia, Netherlands, and Tunisia) had been received to reflect the increasing interest in the topic and the authority in organization of the special issue. Only 16 papers are published with an acceptation rate of $20 \%$. The primary guideline has been the originality of the work, relevance to the topics, and presentation of the contents.

Among the papers of the special issue, a single survey paper and four research papers are devoted to the stabilization and robust control of time-delay systems using Lyapunov theory and LMI tools. Three papers discuss the PID control design problem. Three papers cover industrial and automotive applications using test-benches or simulators to validate 
the proposed approaches. There are also two papers solving synchronization problems and two other papers focusing on control of multiagent systems. Finally, there is a single paper solving the crucial problem of finding solutions for functional differential delayed equations. A very short description of the addressed topics is presented as follows.

The survey paper "Recent Progress in Stability and Stabilization of Systems with Time-Delays," by M. S. Mahmoud, gives an overview of research investigations in the field. All revised results are classified on delay-independent and delay-dependent LMI conditions obtained via LyapunovKrasovskii and Lyapunov-Razumikhin theories where complexity and conservatism of each approach are discussed.

In "Novel Robust Exponential Stability of Markovian Jumping Impulsive Delayed Neural Networks of NeutralType with Stochastic Perturbation," by Y. Fang et al., some new delay-dependent stability conditions are established using Lyapunov-Krasovskii function, Jensen integral inequality, free-weight matrix method, and LMI tools.

In "Robust Stability Criteria for T-S Fuzzy Systems with Time-Varying Delays via Nonquadratic Lyapunov-Krasovskii Functional Approach," by S. H. Kim, a less conservative relaxed condition based on the nonquadratic LyapunovKrasovskii functional is proposed and will be useful for high computational complexities.

In "Robust Quadratic Stabilizability and $H_{\infty}$ Control of Uncertain Linear Discrete-Time Stochastic Systems with State Delay," by X. Jiang et al., a sufficient condition for the existence of a desired robust $H_{\infty}$ controller is obtained.

In "Fixed Points and Exponential Stability for Impulsive Time-Delays BAM Neural Networks via LMI Approach and Contraction Mapping Principle," by R. Rao et al., the authors proposed new LMI-based exponential stability by formulating a contraction mapping in a product space.

In "MIMO PI Controllers for LTI Systems with Multiple Time Delays Based on ILMIs and Sensitivity Functions," by W. Belhaj and O. Boubaker, a temporal/frequency-based design procedure is proposed for synthesis and tuning of MIMO PI controllers for stable, unstable, and nonminimum phase linear systems with delays in state and input variables.

In "Stabilization for Damping Multimachine Power System with Time-Varying Delays and Sector Saturating Actuator," by L. Ma et al., a MIMO PD controller is designed by transforming the problem of PD controller design to that of state feedback stabilizer design for a system in descriptor form. A new sufficient condition is derived based on the Lyapunov theory.

In "An Expert PI Controller with Dead Time Compensation of Monitor AGC in Hot Strip Mill," by F. Zhang et al., the authors presented a monitor automatic gauge control based on hydraulic roll gap control system algorithm with Filtered Smith Predictor suitable for the control of processes with long dead time, simple to implement and tune and having the advantages of obtaining real-time information and improving robustness.

In "A Control Method to Balance the Efficiency and Reliability of a Time-Delayed Pump-Valve System," by Z. Lai et al., a test bench is used to validate the efficiency and the reliability of a sliding mode controller applied to the MIMO
Pump-Valve System by using a Modified Smith Predictor to compensate time-delays of the system.

In "A Decoupling Control Strategy for Multilayer Register System in Printed Electronic Equipment," by S. Liu et al., a control approach based on feedforward control and active disturbance rejection is proposed to solve the strong coupling and strong interference and time-delay problems of multilayer register system used for Printed Electronic Equipment.

In "Direct Yaw-Moment Control of All-Wheel-Independent-Drive Electric Vehicles with Network-Induced Delays through Parameter-Dependent Fuzzy SMC Approach," by W. Cao et al., a robust parameter-dependent fuzzy sliding mode control method based on the real-time information of vehicle states and delays is proposed for all-wheel-independent-drive electric vehicles subject to network-induced delays where the effectiveness of the proposed controller is proved using Simulink and CarSim software.

In "Multiple Model-Based Synchronization Approaches for Time Delayed Slaving Data in a Space Launch Vehicle Tracking System," by H. Song and Y. Choi, the authors give a solution to the serious network delays problem caused by the multiple heterogeneous sensors installed over widespread areas generally leading to the failure of the space launch vehicle tracking systems. They propose a slaving data synchronization approach for the range safety system based on multiple model estimators so that the mission control system can adaptively find an appropriate dynamic model at an arbitrary time index, where time-delays occur.

In "High Precision Clock Bias Prediction Model in Clock Synchronization System," by Z. Liu et al., the authors solve the synchronization problem of the clocks exploited in a distributed system and generally caused by interference time signal transmission or equipment failures. They use the firstorder grey model with one variable optimized using the particle swarm optimization.

In "Consensus Conditions for High-Order Multiagent Systems with Nonuniform Delays," by M. Shi et al., the consensus control problem of third-order to sixth-order multiagent systems with multiple nonuniform time-delays is solved where necessary sufficient conditions are provided in the form of simple inequalities.

In "Consensus Control for a Multiagent System with Time-Delays," by Y. Cao et al., the consensus control problem for a multiagent system of integrator dynamics with input and output time-delays is solved using a state predictor and a linear controller without any delay compensation.

Finally, in "Multiple Periodic Solutions for A Class of Second-Order Neutral Impulsive Functional Differential Equations," by J. Xie et al., the existence solutions of such crucial but frequent problem in modeling many real processes and phenomena is solved by means of critical point theory and variational methods. A typical example is also given to illustrate the applicability of such results.

\section{Acknowledgments}

The editors would like to express their gratefulness to all authors of the special issue for their valuable contributions and to all reviewers for their helpful and professional efforts 
to provide precious comments and feedback. We hope this special issue offers a wide-ranging and timely view of the area of time-delay systems, which will grant stimulation for further academic research and industrial applications.

Olfa Boubaker Valentina E. Balas Abdellah Benzaouia Mohamed Chaabane Magdi S. Mahmoud Quanmin Zhu 


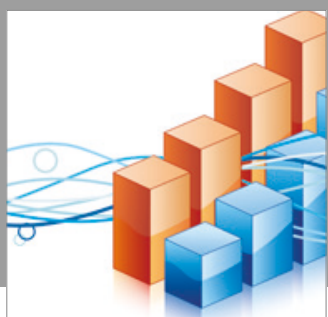

Advances in

Operations Research

vatersals

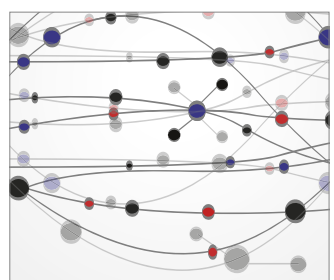

\section{The Scientific} World Journal
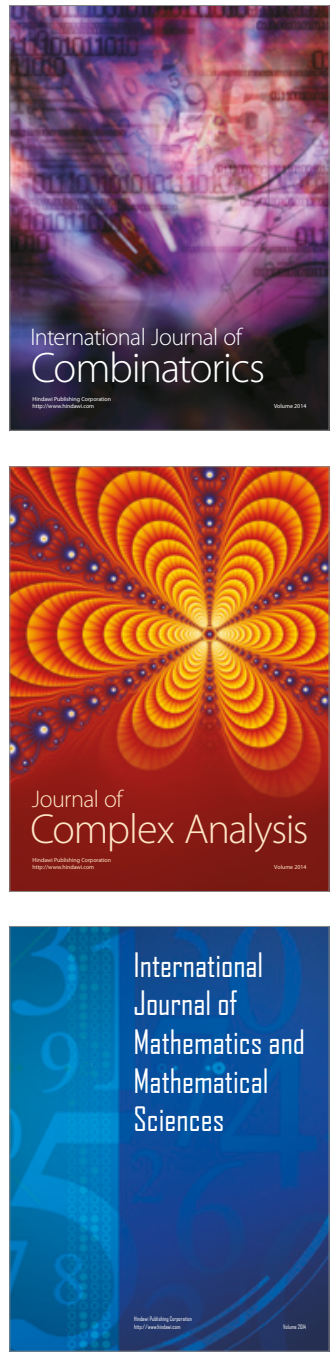
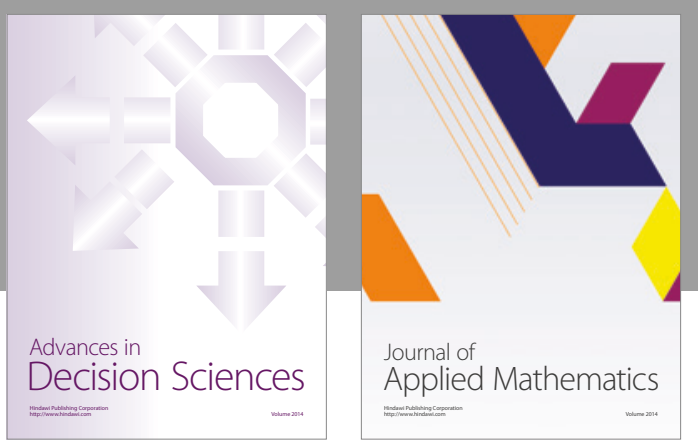

Algebra

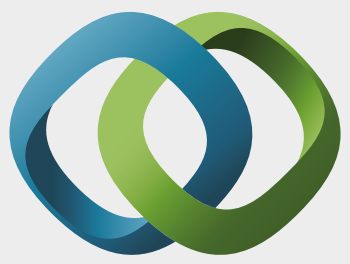

\section{Hindawi}

Submit your manuscripts at

https://www.hindawi.com
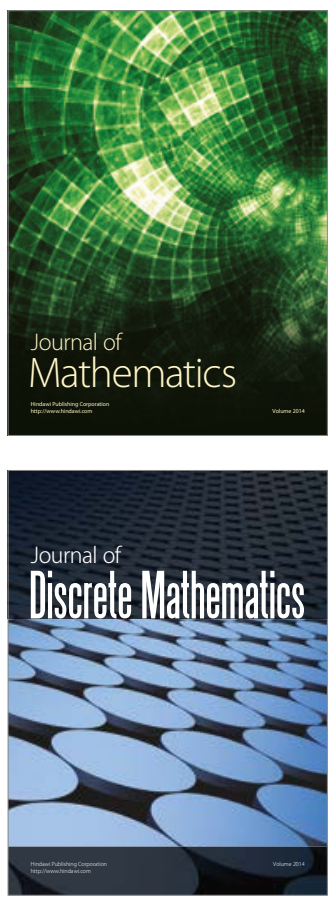

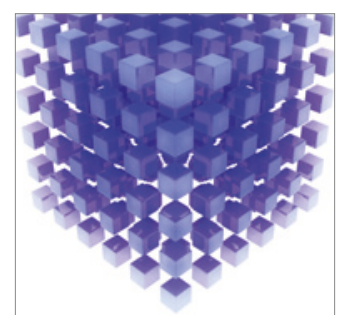

Mathematical Problems in Engineering
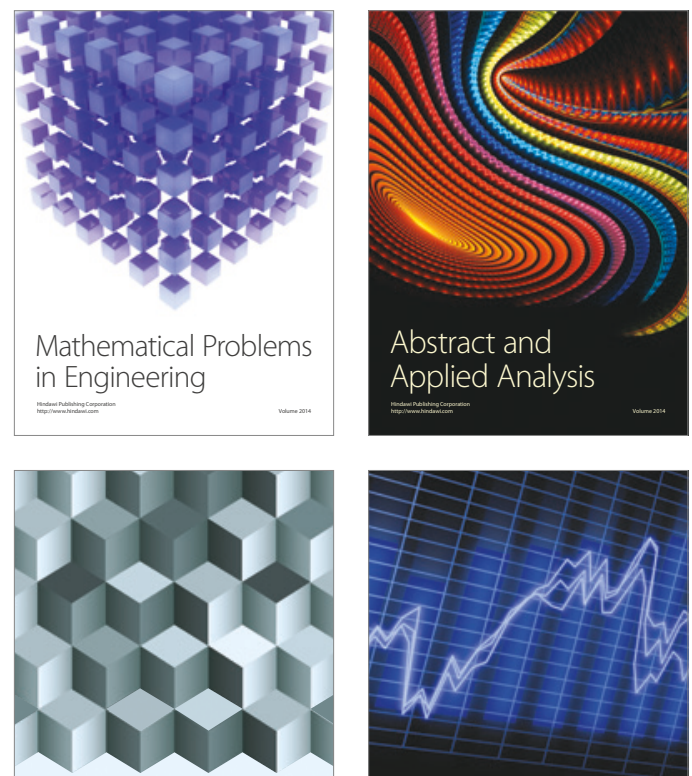

Journal of

Function Spaces

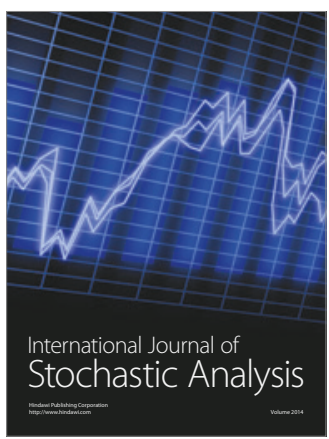

Probability and Statistics
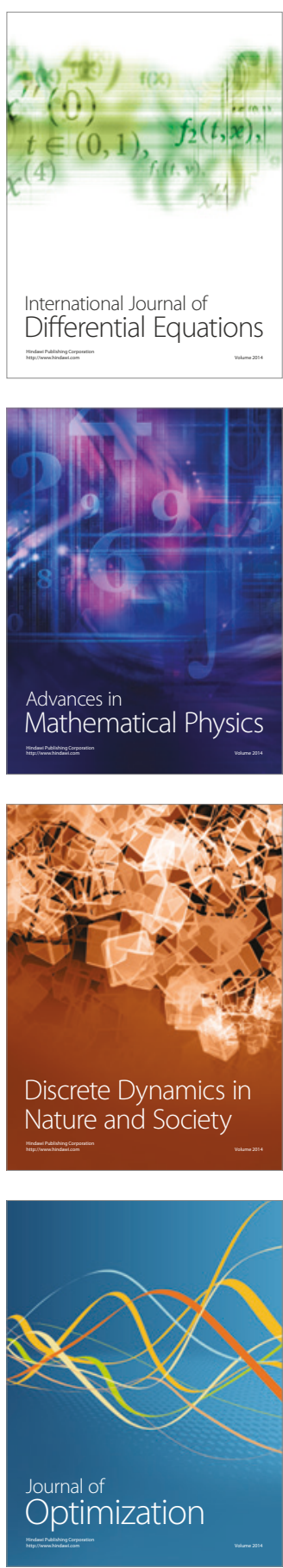